\title{
Some characterizations of Banach lattices with the Schur property
}

\author{
WITOLD WNUK
}

\begin{abstract}
This note contains a short proof of the equivalence of the Schur and Dunford-Pettis properties in the class of discrete KB-spaces. We also present an operator characterization of the Schur property (Theorem 2) and we notice that Banach lattices which are band hereditary $l^{\prime}$ coincide with Banach lattices having the Schur property (this characterization is due to I. Popa [10]). Moreover, the paper offers examples of Banach lattices with the positive Schur property and without the Schur property and which are not isomorphic to any AL-space.
\end{abstract}

\section{INTRODUCTION}

The paper presents a few conditions which are equivalent to the Schur property in the class of Banach lattices (the Schur property means that weakly and norm convergent sequences coincide). These characterizations are due to Varshavskaya and Chuchaev [12] and Popa [10]. Unfortunately, the proof of the characterization offered in [12] (and formulated for some locally convex topologies) contains a mistake. We give another short proof of VarshavskayaChuchaev characterization which points out the coincidence of Schur and Dunford-Pettis properties in some class of Banach lattices (a Banach space $E$ has the Dunford-Pettis property if every weakly compact operator $T: E \rightarrow C_{0}$ is a Dunford-Pettis operator, i.e., $T$ maps relatively weakly compact sets into relatively compact). Moreover, we shall show that it is possible to obtain two characterizations of the Schur property indicated by Popa [10] under somewhat weaker assumptions (we present also slightly different proofs of these characterizations).

The last part of this paper contains a few remarks concerning Banach lattices having the so-called positive Schur property (i.e., $0 \leqslant x_{n} \rightarrow 0$ weakly implies $x_{n} \rightarrow 0$ in norm, see [15]).

1980 Mathematics Subject Classification (1985 revision): 46B30, 47B55, 47D15 Editorial de la Universidad Complutense. Madrid, 1989. 
All notions concerning Banach lattices and not explained here one can find in [2] and [3]. We refer the reader interested in the Dunford-Pettis property to [4] and [3].

Let us only recall the notion of a discrete Riesz space and KB-space which are important in our considerations.

An element $x$ belonging to a Riesz space $E$ is called discrete, if $x>0$ and $|y| \leqslant x$ implies $y=t x$ for some real number $t$ (the above definition, for Dedekind complete Riesz spaces, is equivalent to the following one: if $x=x_{1}+x_{2}$, where $x_{1}, x_{2}$ are positive and disjoint, then $x_{1}=0$ or $x_{2}=0$ ). If every order interval $[0, y]$ in $E$ contains a discrete element, then $E$ is said to be a discrete Riesz space (equivalently: $E$ contains a complete orthogonal system consisting of discrete elements). Roughly speaking, every discrete Banach lattice is a Riesz subspace of some space of sequences (i.e., of the product $R^{x}$-see [2] Theorem 2.17).

A Banach lattice $(E,\|\cdot\|)$ is said to be a $\mathrm{KB}$-space if the norm is order continuous and every increasing norm bounded sequence of positive elements has the supremum in $E$ (equivalently: $\left(E,\|\cdot\|\right.$ ) contains no copy of $c_{0}$ ).

\section{BANACH LATTICES WITH THE SCHUR PROPERTY}

The following lemma, which will be useful in the proof of the first theorem, is a simple consequence of the famous Rosenthal's theorem (see [3] Theorem 14.24$)$.

Lemma (see [11] Theorem 3) For a Banach space $(E,\|\cdot\|)$ the following statements are equivalent:

(i) The dual space $E^{*}$ has the Schur property.

(ii) $E$ has the Dunford-Pettis property and $E$ does not contain an isomorphic copy of $l$.

The theorem mentioned below links Schur and Dunford-Pettis properties in Banach lattices.

Theorem 1. If $(E,\|\cdot\|)$ is a Banach lattice, then the following statements are equivalent:

(i) $E$ is a discrete $K B$-space with Dunford-Pettis property.

(ii) E has the Schur property. 
Proof. (i) $\Rightarrow$ (ii). Let $\left(x_{a}\right)_{a \in A}$ denote a complete orthogonal system in $E$ consisting of discrete elements and denote by $\left(f_{b}\right)$ the positive functionals generated by band projections onto bands $\left\{x_{a}\right\}$ dd. The functionals $f_{a}$ are discrete elements in $E^{*}$ (because they are Riesz homomorphisms). Putting $F=\overline{\operatorname{span}} \mid f_{a}$ : $a \in A\} \subset E^{*}$ we have $F^{*}$ is Riesz isomorphic to $E$ (we can prove this fact using the same method as in a proof that every Banach space with a boundedly complete basis is a dual space). Therefore $F^{*}$ is discrete which implies, together with the Dedekind completeness of $F$ (the space $F$ is an ideal in $E^{*}$ ), that the norm on $F$ is order continuous (see [7] Theorem 2). Thus $F$ does not contain any copy of $l^{\prime}$ ([3] Theorem 12.24). Moreover, $F$ has the Dunford-Pettis property because $F^{*}$ has this property, and so using Lemma we obtain $F^{*}$ has the Schur property and we are done because $F^{*}$ and $E$ are isomorphic.

A proof of the implication (ii) $\Longrightarrow$ (i) is easy and well-known, but for the sake of completeness we shall present it. It is clear that every Banach space with the Schur property has the Dunford-Pettis property. Moreover, a Banach lattice $E$ with the Schur property cannot contain a copy of $c_{0}$, thus our Banach lattice is a KB-space. In particular, the norm has to be order continuous and therefore order intervals are weakly compact. The Schur property implies that order intervals are compact. Hence the Banach lattice $E$ is discrete ([2] Corollary 21.13).

Remark The proof of the Theorem presented in [12] is not correct (even for the case of Banach lattices) because the authors claimed that the following statement holds:

If $H=\overline{\operatorname{span}}\left\{x_{n}: n \in N\right\}$, where $\left(x_{n}\right)$ is a sequence of pairwise disjoint elements in a KB-space $(E,\|\cdot\|)$ satisfying the condition $x_{n} \rightarrow 0$ weakly and $\left\|x_{n}\right\| \geqslant 1$, and $f_{n}$ are biorthogonal functionals associated to the basic sequence $\left(x_{n}\right)$, then $\left(f_{n}\right)$ is not a null sequence in the topology $\sigma\left(F, F^{*}\right)$, where $F=\overline{\operatorname{span}}\left\{f_{n}: n \in N\right\} \subset H^{*}$.

The above statement is false: we have exactly $f_{n} \rightarrow 0$ in the topology $\sigma\left(F, F^{*}\right)$. Indeed, $\left(f_{n}\right)$ is an unconditional basis in $F$ because $f_{n}$ are pairwise disjoint. The basic sequence $\left(x_{n}\right)$ is boundedly complete ( $H$ does not contain a copy of $c_{0}$ ) Thus $H$ has the Radon-Nikodym property ([5] p. 218). Moreover, $H$ and $F^{*}$ are isomorphic ([6] Proposition 1.b.4), and so $F$ contains no copy of $l^{1}([5] \mathrm{p}$. 219). Hence $\left(f_{n}\right)$ is a shrinking basis ([6] Proposition 1.c.9). Let $G \in F^{*}$ and denote $K=\sup _{n}\left\|f_{n}\right\|_{F}\left(K\right.$ is finite because $\left(f_{n}\right)$ is pointiwise convergent). We have

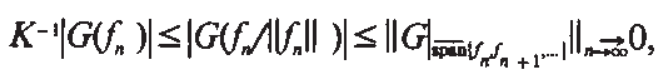

but this means $f_{n} \rightarrow 0$ weakly.

Using Theorem 1 we are able to formulate the following operator characterizations of the Schur property. 
Theorem 2. For a Banach lattice $(E,\|\cdot\|)$ the following statements are equivalent

(i) The norm $\|\cdot\|$ is order continuous and every continuous linear operator $T: E \rightarrow c_{0}$ is a Dunford-Pettis operator.

(ii) $E$ has the Schur property.

(iii) Every positive weakly compact operator $T: l^{l} \rightarrow E$ is compact.

(iv) Every weakly compact operator $T: l^{\prime} \rightarrow E$ is compact.

Proof. (i) $\Rightarrow$ (ii). It is clear that $E$ has the Dunford-Pettis property, Moreover, $E$ is a KB-space. Indeed, if $E$ were not a $\mathrm{KB}$-space then $E$ would contain a complemented copy of $c_{0}$ (because the norm is assumed to be order continuous-see [8]). Let $P: E \rightarrow c_{0}$ be the projection and let $I$ denote the identity operator on $\mathcal{C}_{0}$. Since the composition $I^{\circ} P ; E \rightarrow c_{0}$ is not a Dunford-Pettis operator we have a contradiction.

According to Theorem 1 it is sufficient to show that $E$ is discrete. The order continuity of the norm implies the following fact (see [15] Lemma 1): every Dunford-Pettis operator $T: E \rightarrow c_{0}$ is a difference of two positive operators. Therefore every continuous linear operator from $E$ into $c_{0}$ is regular, and so $E$ has to be discrete $([14])$.

The implication (ii) $\Rightarrow$ (iii) is obvius.

(iii) $\Rightarrow$ (iv). We claim that $(E,\|\cdot\|)$ is a $\mathrm{KB}$-space. In the contrary case $E$ contains a copy of $c_{0}$ and the embedding operator $I: l^{\leftarrow} \rightarrow c_{0} \subset E$ is a positive weakly compact operator which is not compact. Therefore, if $T: l^{\prime} \rightarrow E$ is weakly compact then the modulus $\mid \pi n: l^{1} \rightarrow E$ exists and it is weakly compact too ([3]) Theorem 17.14). The assumption gives that positive operators $|T|+T$ and $|T|$ are compact and thus $T=(|T|+T)-|T|$ is compact.

(iv) $\Rightarrow$ (i). Repeating the argument used in the proof of previous implication we obtain $(E,\|\cdot\|)$ si a KB-space. Moreover, for every continuous linear operator $T: E \rightarrow C_{0}$ and a weakly compact operator $S: l^{\prime} \rightarrow E$ we have $T S$ is compact. Hence $T$ is a Dunford-Pettis operator ([3]) Theorem 19.3).

Remarks. 1. The assumption about order continuity of the norm is essential in (i): every continuous linear operator from $t^{\circ}$ into $c_{0}$ is weakly compact and so it is a Dunford-Pettis operator (because $l^{\circ}$ has the Dunford-Pettis property) but $l^{\infty}$ has not the Schur property.

Similary, the words "every continuous linear operator $T: E \rightarrow c_{0}$ is a Dunford-Pettis operator" cannot be changed by the statement "every positive operator $T: E \rightarrow c_{0}$ is a Dunford-Pettis operator", because $L^{\prime}$ has not the Schur property and $L^{1}$ has the property mentioned. in the statement. 
2. In [10] one can find the following equivalence $(E,\|\cdot\|)$ has the Schur property iff

(a) Every positive weakly compact operator $T: l^{1} \rightarrow E$ is compact and

(b) Every positive operator $T: l^{\infty} \rightarrow E$ is compact.

Theorem 2 shows that (a) implies (b). In other words (a) implies $E$ is discrete and $\|\cdot\|$ is order continuous (compare [9] Theorem 3).

There are known examples of Banach spaces without the Schur property which are hereditary $l^{1}$. In a class of Banach lattices a special kind of a hereditary of $l^{1}$ characterizes the Schur property.

Theorem 3 (see [10]). For a Banach lattice $(E,\|\cdot\|)$ the following statements are equivalent:

(i) E has the Schur property.

(ii) Every closed Banach sublattice contains a band order isomorphic to l.

Proof. (i) $\Rightarrow$ (ii). The space $E$ is a discrete KB-space. Therefore every closed Banach sublattice $F \subset E$ is discrete ([13] Theorem 4). Let $\left(f_{n}\right)$ be a sequence of norm one distinct discrete elements in $F$. According to Rosenthal's theorem we can find a subsequence $\left(f_{n}\right)$ equivalent to the unit vector basis in $l$. Putting $H=\overline{\text { span }}\left\{f_{n_{k}}: k \in N\right\}$ we obtain a required band in $F$.

(ii) $\Rightarrow$ (i). It is clear that $E$ is a $\mathrm{KB}$-space because $E$ cannot contain an order copy of $c_{0}$. Moreover, $E$ is discrete. Indeed, if $0<x \in E$ and $B=\{x\}^{d d}$ then $B=H \oplus H^{d}$ where $H$ is a band order isomorphic to $l^{1}$. Let $P$ denote the band projection of $B$ onto $H$. We have $P x>0$ and so there is a discrete element $y$ in $H$ such that $0<y \leqslant P x \leqslant x$. Since every discrete element in $H$ is discrete in $E$ then we obtain that every order interval in $E$ contains discrete element, i.e., $E$ is discrete.

Let $\mathrm{A}$ be a relatively weakly compact subset of $E$. According to [3] Theorem 13.8 the set sol $A$, i.e, the solid hull of $A$, is also relatively weakly compact. We claim that

$\left(^{*}\right)\left\|x_{n}\right\| \rightarrow 0$ for an arbitrary sequence $\left(x_{n}\right)$ of pairwise disjoint elements belonging to sol $A$.

Indeed, suppose $\mathrm{c}=\inf _{\mathrm{n}}\left\|x_{n}\right\|>0$ for some sequence of disjoint elements from sol $A$. Since sol $A$ is relatively weakly compact, then there exists a subsequence $\left(x_{n_{k}}\right)$ which is weakly null. Using [2] Theorem 21.14 we obtain that $\left(x_{n}\right)$ is weakly null too. The sublattice $\overline{\operatorname{span}}\left\{\left|x_{n}\right|: k \in N\right\}$ contains a band isomorphic to $l^{l}$, and so span $\left\{x_{\mathrm{n}} \mid: i \in N\right\}$ is isomorphic to $l^{l}$ for some subsequence $\left(\left|x_{n_{k_{i}}}\right|\right)$. Therefore $\left\|x_{n_{k_{i}}}\right\| \rightarrow 0$ anld we have a contradiction because $\left\|x_{n_{k_{i}}}\right\| \geqslant c$. 
According to [2] Theorem 21.15 the condition $\left(^{*}\right)$ implies relative compatness of the sol $A$. Thus $E$ has the Schur property.

Remark. In [10] the statement (ii) contains an additional assumption that $E$ is discrete.

\section{REMARKS ON BANACH LATTICES WITH THE POSITIVE SCHUR PROPERTY}

Besides the Schur property one can consider a weaker property called the positive Schur property ([15]), i.e., $0 \leq x_{n} \rightarrow 0$ weakly implies $x_{n} \rightarrow 0$ in norm. A simple example of a Banach lattice with the positive Schur property and without the Schur property is a space $L^{\prime}(\mu)$, where $\mu$ is not purely atomic.

The following construction produces a family of Banach lattices with the positive Schur property. Let $\lambda$ be an order dense ideal in the product $R^{N}$ with the coordinatewise order. Suppose $p$ is a norm on $\lambda$ such that $(\lambda, p)$ is a Banach lattice with the Schur property. If $\lambda\left(\mathrm{L}^{1}\right)=\left\{\left(f_{n}\right) \subset L^{\prime}(0,1):\left(\left\|f_{n}\right\|\right)_{n=1}^{\infty} \in \lambda\right\}$, then the pair $\left(\lambda\left(L^{y}\right), \hat{p}\right)$ is a Banach lattice with the positive Schur property, where $\hat{p}\left(\left(f_{n}\right)\right)=p\left(\left(\left\|f_{n}\right\|\right)\right)$.

Indeed, an arbitrary sequence $\left(a_{n}\right)$ belonging to the Köthe dual of $\lambda$ determines a continuous linear functional $G$ on $\lambda\left(L^{\prime}\right)$ :

$$
G\left(\left(f_{n}\right)\right)=\sum_{n=1}^{\infty} a_{n} \int_{0}^{1} f_{n} d t
$$

Therefore, if $\left(F_{n}\right) \subset \lambda\left(L^{\prime}\right)$ is a weakly null sequence with positive terms (i.e., $F_{n}=\left(f_{n}(k)\right)_{k=1}^{\infty}$, and $f_{n}(k) \geqslant 0$ for all $\left.n, k\right)$, then $G\left(\left(F_{n}\right)\right)=\sum_{n=1}^{\infty} a_{k}\left\|f_{n}(k)\right\| \rightarrow 0$ as $n \rightarrow \infty$. This means that $x_{n}=\left(\left\|f_{n}(k)\right\|_{n \rightarrow \infty} \rightarrow 0\right.$ weakly in $\lambda$, and so $p\left(x_{n}\right)$ tends to zero. In other words $\hat{p}\left(F_{n}\right) \rightarrow 0$. It is clear that $\lambda\left(L^{\prime}\right)$ has not the Schur property.

It is worth to notice the following fact: if $\lambda$ is not isomorphic to $l^{1}$, then $\lambda\left(L^{\prime}\right)$ is not isomorphic to any $L^{\prime}(\mu)$. We claim that if $\lambda\left(L^{\prime}\right)$ were isomorphic to $L^{\prime}(\mu)$ for some measure $\mu$, then it would be isomorphic to $L^{\prime}(0,1)$.

Indeed, since $\lambda\left(L^{1}\right)$ is separable, then $\mu$ has to be separable and so $\sigma$-finite, in particular. Therefore $\lambda\left(L^{\prime}\right)$ is isomorphic to $L^{\prime}(v)$ for some probabilistic measure $v$. The space $L^{\prime}(v)$ is isomorphic to $L^{\prime}(v) \oplus l^{1}$, where $v_{c}$ is the atomless part of $v$. According to the famous Caractheodory's theorem $L^{\prime}\left(v_{c}\right)$ is isomorphic to $L^{\prime}(0,1)$. Finally, $L^{\prime}(v)$ is isomorphic to $L^{\prime}(0,1)$. The Banach lattice $\lambda\left(L^{1}\right)$ contains no discrete elements, and so $\lambda\left(L^{1}\right)$ and $L^{1}(0,1)$ are order isomorphic in virtue of Abramovic -Wojtaszczyk result ([1] Theorem 2). Since $\lambda$ is a sublat- 
tice of $\lambda\left(L^{1}\right)$ and $\lambda\left(L^{1}\right)$ is order isomorphic to $L^{1}(0,1)$, then $\lambda$ has to be isomorphic to $l^{l}-$ a contradiction.

We can also notice that using the above proof and another result of Abramovič and Wojtaszczyk ([1], Theorem 5) we have that if $E$ is a separable $\sigma$-Dedekind complete Banach lattice isomorphic to an $\mathrm{AL}$-space, then $E$ is order isomorphic to an AL-space.

It is known that for discrete Banach lattices the positive Schur property and the Schur property coincide (see [15]).

The positive Schur property has the following characterization

Theorem 4 (see [15]). For a Banach lattice $(E,\|\cdot\|)$ the following statements are equivalent:

(i) $E$ is $\sigma$-Dedekind complete and it has the property

$\left(^{*}\right)$ An operator $T: E \rightarrow c_{0}$ is a Dunford-Pettis operator iff $T$ is a difference of two positive operators from $E$ into $c_{0}$.

(ii) E has the positive Schur property.

One can easly verify that a Banach lattice has the positive Schur property iff every sequence of norm one positive pairwise disjoint elements contains a subsequence equivalent to the unit vectors in $l^{l}$ (see [15]).

\section{References}

[1] JU. A. ABRAMOVIC and P. WoJTASZCZYK, The uniqueness of the order in the spaces $L[0,1]$ and $l^{p}$ (Russian), Mat. Zametki 18 (1975), 313-325.

[2] C. Aliprantis and O. Burkinshaw, Locally solid Riesz spaces, Pure and Applied Mathematics Series No 76, Academic Press, New York and London 1978.

[3] C. Aliprantis and O. Burkinshaw, Positive, operators, Pure and Applied Mathematics Series, Academic Press, New York and London 1985.

[4] J. DIESTEL, A survey of results related to the Dunford-Pettis property, Integration, topology and geometry in linear spaces, Proc. Conf. Chapel Hill N.C. 1979, Cotemp. Math. 2, 15-60 (1980).

[5] J. Diestel and J.J. UHL, Vector measures, Math. Surveys, No 15, Amer, Math. Soc. 1977.

[6] J. Lindenstrauss and L. TZAFrirI, Classical Banach spaces I, Sequence spaces. Ergebnisse 92, Berlin-Heidelberg-New York: Springer Verlag 1977.

[7] G. JA. Lozanovskiī, Discrete functionals in Marcinkiewicz and Orlicz spaces (Russian), Studies in the theory of functions of several real 
variables, No. 2 (Russian), pp. 132-147, Jaroslav. Gos. Univ., Yaroslavl, 1978.

[8] P. MEYER-NIEBERG, Charakterisierung einiger topologischer und ordungstheoretischer Eigenschaften von Banachverbanden mit Hilfe disjunkter Folgen, Arch. Math. 24 (1973), 640-647.

[9] I. POPA, Propriétés opérationnelles des spaces de Banach réticulés et discrets, C. R. Acad. Sc. Paris 283 (1976), 1013-1015.

[10] I. POPA, Espaces de Banach réticulés ayant la propriété de Schur, C.R. Acad. Sc. Paris, 285 (1977), 629-631.

[11] P. PETHE and N. THAKARE, Note on Dunford-Pettis property and Schur property, Indiana Math. J. 27 (1978), 91-92.

[12] E. V. VARShAVSKAYA and I.I. ChUCHAEV, On the Dunford-Pettis and Schur properties in locally convex $K$-spaces (Russian), Soobsc. Akad. Nauk Gruzin. SSR 120 (1985), 21-23.

[13] W. WNUK, When is the closure of an atomic Riesz subspace atomic?, Bull. Ac. Pol. Math. 34 (1986), 669-694.

[14] W. WNUK, A characterization of discrete Banach lattices with order continuous norms, Proc. Amer. Math. Soc. 104 (1988), 197-200.

[15] W. WNUK, A note on the positive Schur property, Glasgow Math. J. 31 (1989), 169-172.

Mathematical Institute

Polish Academy of Sciences

Poznań Branch

Mielzyńskiego 27/29

61-725 Poznan (POLAND) 